\title{
Coincidence of bft and cfiA genes in a multi-resistant clinical isolate of Bacteroides fragilis
}

Although a common member of the normal human gut flora, Bacteroides fragilis possesses various virulence factors, such as capsule, LPS and special surface proteins, and certain types of strains may produce an extracellular enterotoxin, termed fragilysin (BFT). The fragilysinencoding gene $(b f t)$ has three different isoforms (termed $b f t-1, b f t-2$ and $b f t-3$ ) (Chung et al., 1999; Franco et al., 1997; Kling et al., 1997). Because of the increasing number of resistant $B$. fragilis isolates, the therapeutic possibilities have been restricted to only some antibiotics, such as piperacillin/tazobactam, imipenem, metronidazole, clindamycin and cefoxitin (Rasmussen et al., 1993). Out of these, metronidazole and imipenem are the drugs of choice in the treatment of infections caused by $B$. fragilis; however, the emergence of imipenem or metronidazole resistant isolates has been reported (Rasmussen et al., 1993). Carbapenem and metronidazole resistances are frequently associated with the presence of the $c f i A$ gene encoded class B metallo- $\beta$-lactamase (Thompson \& Malamy, 1990) or various types of 5-nitroimidazole resistance genes ( $\operatorname{nim} A-G$ ) (Gal \& Brazier, 2004). Strains carrying the cfiA gene may show susceptibility to imipenem, because in most isolates this gene is silent, but the presence of insertion (IS) elements in the upstream region of this gene can lead to the development of carbapenem resistance (Podglajen et al., 1992).

By using various molecular typing methods, such as arbitrarily primed-PCR, PFGE and multilocus enzyme electrophoresis, $b f t$-positive, $c f i A$-negative strains and $b f t$-negative, $c f i A$-positive strains belonged to two different DNA homology groups, and the coexistence of $b f t$ and $c f i A$ genes was not detected (Gutacker et al., 2000; Vallim et al., 2002).

During examination of the prevalence of $b f t$ - or $c f i A$-positive $B$. fragilis strains isolated from various clinical specimens, we found a multi-resistant $B$. fragilis strain R19811 isolated from a blood culture and identified by rDNA RFLPs in the Anaerobe Reference Laboratory, Cardiff (Wareham et al., 2005). This strain co-harboured the $c f i A$ and $b f t$ genes; therefore, our aim was to characterize its genetic background.

B. fragilis strain R19811 and control strains were cultured as previously described (Sóki et al., 2000). The list and the description of the control strains have been described before (Sóki et al., 2006).

MICs to a range of antibiotics were determined by using the E-test method (AB Biodisk) according to the manufacturer's instructions. B. fragilis strain R19811 was multi-resistant to penicillin $\mathrm{G}\left(\mathrm{MIC} \geqslant 256 \mu \mathrm{g} \mathrm{ml}^{-1}\right)$, amoxicillin/clavulanic acid (MIC $\geqslant 256 \mu \mathrm{g}$ $\left.\mathrm{ml}^{-1}\right)$, cefoxitin $\left(\mathrm{MIC} \geqslant 256 \mu \mathrm{g} \mathrm{ml}^{-1}\right.$ ), imipenem $\left(\mathrm{MIC} \geqslant 256 \mu \mathrm{g} \mathrm{ml}^{-1}\right.$ ), clindamycin $\left(\mathrm{MIC} \geqslant 256 \mu \mathrm{g} \mathrm{ml}^{-1}\right.$ ) and metronidazole $\left(\mathrm{MIC}=64 \mu \mathrm{g} \mathrm{ml}^{-1}\right.$ ). Because of the imipenem resistance, the presence of $c f i A$ gene encoding metallo- $\beta$ lactamase was tested. $c f i A$ PCR (Podglajen et al., 1992; Thompson \& Malamy, 1990) revealed that the strain carried the cfiA gene, at the same time the presence of $b f t$ gene was also detected by PCR according to Pantosti et al. (1997).

We analysed the upstream region of the cfiA gene to detect IS elements, hypothesizing that the occurrence of one of these sequences may contribute to the development of carbapenem resistance mechanisms; in B. fragilis strain R19811 an insertion was detected in the upstream region of $c f i A$ gene by $\mathrm{G}$ and $\mathrm{Up} 2$ primers, as described by Podglajen et al. (1994). To determine the type of this element, various primers, IS614 (IS614-1i, 5'-GAAATTGTGTATCAATGCCG-3' and IS614-2i, 5' -CTGATACCATCCTCAGAGCC-3'), IS942 (Rasmussen \& Kovacs, 1991), IS 1169 (Trinh et al., 1995), IS1170/1 and IS1170/2 (Trinh et al., 1995), IS 1186 (Podglajen et al., 1994), and IS4351
(Podglajen et al., 1994; Rasmussen et al., 1987) were tested for use in PCR. During these amplification reactions, standard PCR reaction mixture was used, as described previously by Sóki et al. (2006). Of the examined IS elements, only IS614 was detected by PCR. Partial nucleotide sequence data of the upstream region of the $c f i A$ gene by Up2, G, IS614-1i and IS614-2i primers, as described by Sóki et al. (2004), confirmed that it is an IS614B element.

B. fragilis strain R19811 contained plasmids with an estimated molecular mass of 5.6 and $9.9 \mathrm{~kb}$ using HindIII restriction enzyme (Pharmacia Biotech) analysis. In spite of the fact that $c f i A$ gene could be on a plasmid (Bandoh et al., 1992; Nakano et al., 2004), in our case, Southern blot hybridization, showed it was not.

In spite of the observed metronidazole resistance, nim genes commonly responsible for metronidazole resistance in the $B$. fragilis group could not be detected using the nim-3 and nim-5 primer pair (Trinh \& Reysset, 1996), which is suitable for the detection for all described nim genes. The annealing temperature of nim PCR was reduced from 62 to $52{ }^{\circ} \mathrm{C}$ to show incidental sequence variation in the nim gene, but PCR products could not be detected. Further investigations are necessary to establish the metronidazole resistance mechanism in this organism.

In numerous earlier studies, the presence of the $c f i A$ gene was never observed together with the cepA gene encoding endogenous class A $\beta$-lactamase, because $c f i A$-positive and $с е p A$-positive strains belong to two genetically distinct groups. The first group was characterized by the presence of $c f i A$ gene, while in the second group, the presence of the cepA gene is typical with or without the presence of the bft gene. In spite of this observation, in 2005, Ayala et al. (2005) described two B. fragilis strains (7160 and 213E), which 


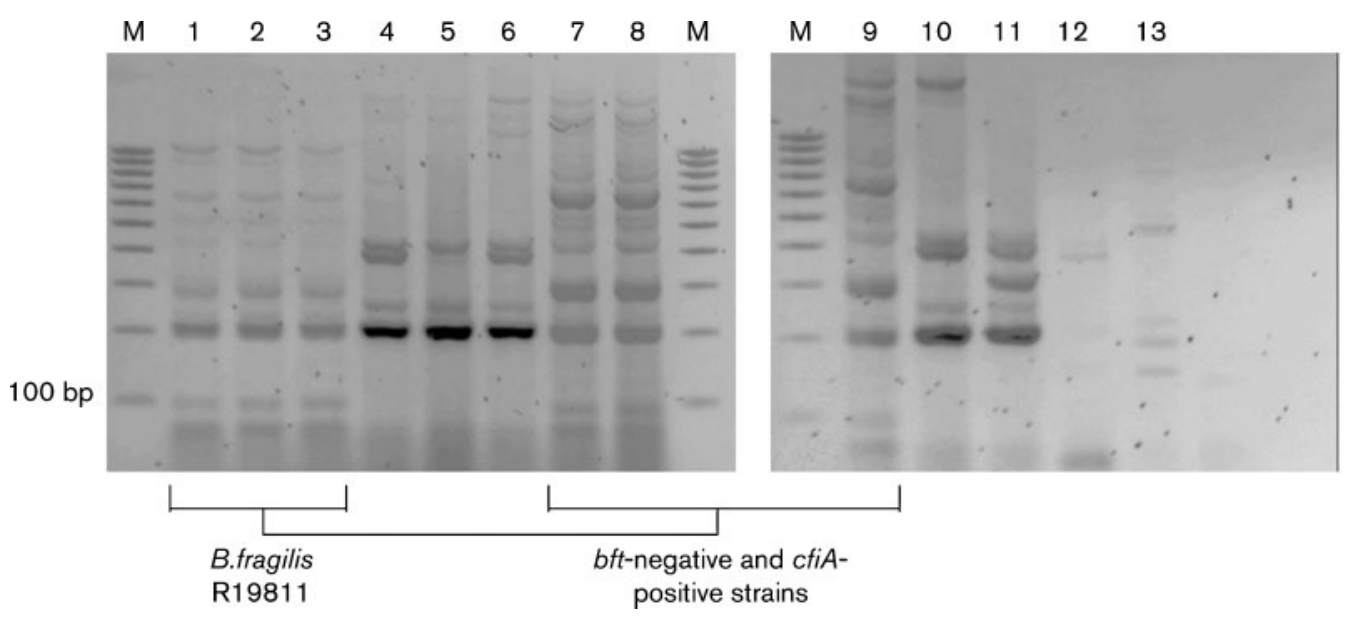

Fig. 1. Comparative ERIC1-2 PCR pattern of $B$. fragilis strain R19811. Two groups of $B$. fragilis strains with similar patterns are indicated: the cfiA- and bft-positive strain R19811 in triplicate (first group) and bft-negative, cfiA-positive strains (second group). Lanes 1-3, cfiA- and bft-positive $B$. fragilis strain R19811; lanes 4-6, bft-positive and cfiA-negative isolates; lanes 7-9, bft-negative strains and cfiA-positive strains; lanes 10-11, bft-negative and cfiA-negative $B$. fragilis strains; lane 12, Bacteroides thetaiotaomicron; lane 13, 'Bacteroides variabilis'. M, 100 bp ladder (Sigma; 100-1000 bp).

possess both the cepA and the $c f i A$ gene. In strain R19811, no amplification product was detected with cepA PCR as described by Gutacker et al. (2000). This suggests that the strain originally belonged to the cfiA-positive group.

In the cytotoxicity assay, as described by Pantosti et al. (1994), B. fragilis strain R19811 caused a reversible cytopathic effect on the HT-29 cell line, this result indicated that in the cell culture supernatant, functional toxin, namely fragilysin was present. PCR-RFLP analysis of the $b f t$ gene by BTT1 (5' -CATGTTCTAATGAAGCTGATTC-3') and BTT2 (5'-ATCGCCATCTGCTGTTTCCC- $3^{\prime}$ ) primers, with minor modification according to Chung et al. (1999), revealed a $b f t-1$ allele.

To clarify the epidemiological background of this strain, enterobacterial repetitive intergenic consensus (ERIC) PCR typing (Versalovic et al., 1991) was applied in the case of $b f t$-positive and $c f i A$-negative, $b f t$-negative and $c f i A$-positive, $b f t$ - and cfiA-negative strains, and the PCR patterns were compared with the result of ERIC PCR in B. fragilis strain R19811. Two homology groups of $B$. fragilis can be distinguished by using molecular typing methods as described previously by Gutacker et al. (2000), Moraes et al. (1999) and Ruimy et al. (1996): the first group is characterized by the presence of the $c f i A$ gene, these strains were $b f t$-negative, while the strains in the second group carried only the bft gene (Fig. 1). ERIC PCR typing suggested that strain R19811, which harboured the bft and cfiA gene simultaneously, may be related to the first group, which contained only $c f i A$-positive strains (Fig. 1).

In summary, analysis of the results of cfiA and cepA PCR, and ERIC typing pattern, indicated that $B$. fragilis strain 19811 originally carried the $c f i A$ gene and acquired the bft gene only later. Franco (2004) described two new related conjugative transposons (CTn86 and CTn9343) in connection with the bft gene. Recently, a more divergent picture has arisen. The two conjugative transposons differ in two regions: one, BfPAI, found exclusively on CTn86 and harbouring the $b f t$ genes, and the other exclusively harbouring an additional $7 \mathrm{~kb}$ region and found on CTn9343. However, these two additional regions with the leftend of the transposons may result in hybrid elements. Still, the presence of CTn86 is mainly characteristic for $b f t$ genes and the pattern I of $B$. fragilis strains, while CTn9343 and the CTn9343like elements mainly result in pattern III. The $c f i A$ gene, however, was found only in pattern II and some pattern III isolates, but still in division II (Buckwold et al., 2007). If genes responsible for the transfer of these CTns are functional, it is possible that the bft gene can be transferred from an enterotoxigenic strain to a nontoxigenic strain. The presence of new transmissible CTns, and virulence and resistance genes, may contribute to the enhancement of the pathogenicity potential and the fitness of this strain; therefore, the aforementioned factors may promote the development of more severe infection, which may be unresponsive to the majority of the therapeutic possibilities.

\section{Acknowledgements}

This work was supported by grants KMA0304 and T037475 from Ministry of Economy and Transport, Hungary and the Hungarian National Research Foundation, respectively.

\section{Gabriella Terhes, ${ }^{1}$ Jon S. Brazier, ${ }^{2}$ József Sóki, ${ }^{1}$ Edit Urbán ${ }^{1}$ and Elisabeth Nagy ${ }^{1}$}

\footnotetext{
${ }^{1}$ Department of Clinical Microbiology, Faculty of Medicine, University of Szeged, H-6725 Szeged, Hungary

${ }^{2}$ Anaerobe Reference Laboratory, NPHS Microbiology Cardiff, University Hospital of Wales, Cardiff, UK
}

Correspondence: Elisabeth Nagy
(nagye@mlab.szote.u-szeged.hu) 
Ayala, J., Quesada, A., Vadillo, S., Criado, J. \& Piriz, S. (2005). Penicillin-binding proteins of Bacteroides fragilis and their role in the resistance to imipenem of clinical isolates. J Med Microbiol 54, 1055-1064.

Bandoh, K., Watanabe, K., Muto, Y., Kato, N. \& Ueno, K. (1992). Conjugal transfer of imipenem resistance in Bacteroides fragilis. J Antibiot 45, 542-547.

Buckwold, S. L., Shoemaker, N. B., Sears, C. L. \& Franco, A. A. (2007). Identification and characterization of conjugative transposons CTn86 and CTn9343 in Bacteroides fragilis strains. Appl Environ Microbiol 73, 53-63.

Chung, G. T., Franco, A. A., Wu, S., Rhie, G., Cheng, R., Oh, H. \& Sears, C. L. (1999). Identification of a third metalloprotease toxin gene in extraintestinal isolates of Bacteroides fragilis. Infect Immun 67, 4945-4949.

Franco, A. A. (2004). The Bacteroides fragilis pathogenicity island is contained in a putative novel conjugative transposon. J Bacteriol 186, 6077-6092.

Franco, A. A., Mundy, L. M., Trucksis, M., Wu, S. Kaper, J. B. \& Sears, C. L. (1997). Cloning and characterization of the Bacteroides fragilis metalloprotease toxin gene. Infect Immun 65, 1007-1013

Gal, M. \& Brazier, J. S. (2004). Metronidazole resistance in Bacteroides spp. carrying nim genes and the selection of slow-growing metronidazole-resistant mutants. J Antimicrob Chemother 54, 109-116.

Gutacker, M., Valsangiacomo, C. \& Piffaretti, J. C. (2000). Identification of two genetic groups in Bacteroides fragilis by multilocus enzyme electrophoresis: distribution of antibiotic resistance $(c f i A, c e p A)$ and enterotoxin $(b f t)$ encoding genes. Microbiology 146, 1241-1254.

Kling, J. J., Wright, R. L., Moncrief, J. S. \& Wilkins, T. D. (1997). Cloning and characterization of the gene for the metalloprotease enterotoxin of Bacteroides fragilis. FEMS Microbiol Lett 146, 279-284.

Moraes, S. R., Goncalves, R. B., Mouton, C., Seldin, L., Ferreira, M. C. \& Domingues, R. M.
(1999). Bacteroides fragilis isolates compared by AP-PCR. Res Microbiol 150, 257-263.

Nakano, V., Padilla, G., do Valle Marques, M. \& Avila-Campos, M. J. (2004). Plasmid-related $\beta$-lactamase production in Bacteroides fragilis strains. Res Microbiol 155, 843-846.

Pantosti, A., Cerquetti, M., Colangeli, R. \& D’Ambrosio, F. (1994). Detection of intestinal and extra-intestinal strains of enterotoxigenic Bacteroides fragilis by the HT-29 cytotoxicity assay. J Med Microbiol 41, 191-196.

Pantosti, A., Malpeli, M., Wilks, M., Grazia Menozzi, M. \& D'Ambrosio, F. (1997). Detection of enterotoxigenic Bacteroides fragilis by PCR. J Clin Microbiol 35, 2482-2486.

Podglajen, I., Breuil, J., Bordon, F., Gutmann, L. \& Collatz, E. (1992). A silent carbapenemase gene in strains of Bacteriodes fragilis can be expressed after a one-step mutation. FEMS Microbiol Lett 70, 21-29.

Podglajen, I., Breuil, J. \& Collatz, E. (1994). Insertion of a novel DNA sequence, IS1186, upstream of the silent carbapenemase gene $c f i A$, promotes expression of carbapenem resistance in clinical isolates of Bacteroides fragilis. Mol Microbiol 12, 105-114.

Rasmussen, B. A. \& Kovacs, E. (1991). Identification and DNA sequence of a new Bacteroides fragilis insertion sequence-like element. Plasmid 25, 141-144.

Rasmussen, J. L., Odelson, D. A. \& Macrina, F. L. (1987). Complete nucleotide sequence of insertion element IS4351 from Bacteroides fragilis. J Bacteriol 169, 3573-3580.

Rasmussen, B. A., Bush, K. \& Tally, F. P. (1993). Antimicrobial resistance in Bacteroides. Clin Infect Dis 16 (Suppl.), S390-S400.

Ruimy, R., Podglajen, I., Breuil, J., Christen, R. \& Collatz, E. (1996). A recent fixation of $c f i A$ genes in a monophyletic cluster of Bacteroides fragilis is correlated with the presence of multiple insertion elements. J Bacteriol 178, 1914-1918.

Sóki, J., Urbán, E., Szöke, I., Fodor, E. \& Nagy, E. (2000). Prevalence of the carbapenemase gene (cfiA) among clinical and normal flora isolates of Bacteroides species in Hungary. J Med Microbiol 49, 427-430.

Sóki, J., Fodor, E., Hecht, D. W., Edwards, R., Rotimi, V. O., Kerekes, l., Urbán, E. \& Nagy, E. (2004). Molecular characterization of imipenem-resistant, cfiA-positive Bacteroides fragilis isolates from the USA, Hungary and Kuwait. J Med Microbiol 53, 413-419.

Sóki, J., Gal, M., Brazier, J. S., Rotimi, V. O., Urbán, E., Nagy, E. \& Duerden, B. I. (2006).

Molecular investigation of genetic elements contributing to metronidazole resistance in Bacteroides strains. J Antimicrob Chemother 57, 212-220.

Thompson, J. S. \& Malamy, M. H. (1990). Sequencing the gene for an imipenem-cefoxitinhydrolyzing enzyme (CfiA) from Bacteroides fragilis TAL2480 reveals strong similarity between CfiA and Bacillus cereus $\beta$-lactamase II. $J$ Bacteriol 172, 2584-2593.

Trinh, S. \& Reysset, G. (1996). Detection by PCR of the nim genes encoding 5nitroimidazole resistance in Bacteroides spp. J Clin Microbiol 34, 2078-2084.

Trinh, S., Haggoud, A., Reysset, G. \& Sebald, M. (1995). Plasmids pIP419 and pIP421 from Bacteroides: 5-nitroimidazole resistance genes and their upstream insertion sequence elements. Microbiology 141, 927-935.

Vallim, D. C., Oliveira, I. C. M., Antunes, E. N. F., Ferreira, E. O., Moraes, S. R., Paula, G. R., SilvaCarvalho, M. C., Figueiredo, A. M. S., Ferrreira, M. C. S. \& Domingues, R. M. C. P. (2002). Evaluation of genetic relatedness of Bacteroides fragilis strains isolated from different sources by AP-PCR and pulsed-field gel electrophoresis assay. Anaerobe 8, 192-199.

Versalovic, J., Koeuth, T. \& Lupski, R. (1991). Distribution of repetitive DNA sequences in eubacteria and application to fingerprinting of bacterial genomes. Nucleic Acids Res 19, 6823-6831.

Wareham, D. W., Wilks, M., Ahmed, D., Brazier, J. S. \& Millar, M. (2005). Anaerobic sepsis due to multidrug resistant Bacteroides fragilis: microbiological cure and clinical response with linezolid therapy. Clin Infect Dis 40, e67-e68. 